\title{
A physical basis for Hubbert's decline from the midpoint empirical model of oil production
}

\author{
R. J. Wiener ${ }^{1} \&$ D. M. Abrams ${ }^{2}$ \\ ${ }^{1}$ Research Corporation, USA \\ ${ }^{2}$ Department of Earth, Atmospheric, and Planetary Science, MIT, USA
}

\begin{abstract}
Just over fifty years ago, Hubbert correctly predicted that US oil production in the lower-48 states would soon peak, despite substantially rising production at the time. Hubbert based his prediction on the empirical observation that the production rate at oil reservoirs typically follows a bell-shaped curve that peaks near the midpoint of production. We present a highly simplified physical model of oil production that gives insight into Hubbert's success. This toy model relates the total area of active wells to future production and allows for several interesting analytical conclusions. We show analytically, contrary to intuition but consistent with historical data, the number of active wells at any given field must peak after the production rate peaks. Since oil production is pressuredriven, the toy model treats an oil reservoir as a sealed container filled with liquid oil and highly pressurized gas. A pipe extends into the container with a cross-sectional area, which represents the total area of wells and changes continuously over time. As gas expands, it forces liquid out of the container through the pipe but also drops in pressure, eventually limiting production. We assume the flow obeys Bernoulli's principle and the gas expands isothermally, which leads to a nonlinear ordinary differential equation for the velocity of fluid exiting the container. For most reasonable choices of the area function, the differential equation requires a numerical solution. However, it is possible to specify a plausible area function for which the model yields an analytical expression for the production rate that corresponds to the bell-shaped curve used by Hubbert to predict the peak in US oil production.
\end{abstract}

Keywords: Hubbert's peak, oil production, logistic growth, pressure-driven flow. 


\section{Introduction}

In 1956, Hubbert correctly predicted US oil production in the lower-48 states would peak around 1970 [1]. Many oil recovery experts greeted Hubbert's prediction with scepticism [2], since it came at a time when oil production in the lower-48 states was rising substantially. Hubbert based his prediction on the simple empirical observation that the peak production rate at oilfields $(e . g$. in units of barrels per day), or more broadly in oil producing regions, typically occurs at about the midpoint in production, i.e. at the time when half the recoverable oil has been produced. Hubbert observed that typically a sigmoidshaped function is a good approximation to data for the total volume of oil recovered from an oil reservoir. The solution to the logistic differential equation is an example of such a function. The derivative of this function with respect to time then corresponds to the oil production rate and is a bell-shaped curve. Thus, by fitting data for the oil production rate in the lower-48 states to a function that has the form of the derivative of the solution to the logistic differential equation, Hubbert was able to extrapolate the curve and correctly predict its peak would occur around 1970.

In this paper, we develop a highly simplified physical model of oil production, in effect a "toy" model, which elucidates underlying physical aspects of oil production and helps explain Hubbert's success. The toy model relates the total cross-sectional area of active wells to future production. We show analytically the model is equivalent to the logistic differential equation under plausible assumptions about how the area of producing wells typically varies over time. We also show analytically, contrary to intuition, the number of active wells at any given field must peak after the production rate peaks. We confirm this counter-intuitive consequence of the model with historical data from major oilfields on four continents.

\section{Logistic growth applied to oil production}

Hubbert pioneered the idea of using logistic growth to model oil production [1-3]. The logistic growth function satisfies the logistic differential equation $\dot{Q}=r\left(1-Q / Q_{t o t}\right) Q$, where $Q$ is the quantity that is growing, $\dot{Q}$ is the derivative of $Q$ with respect to time, $r$ is the initial rate of growth, and $Q_{t o t}$ is the value to which $Q$ is asymptotically growing. Logistic growth describes any growth process in which the per capita growth rate, $\dot{Q} / Q$, decreases linearly as $Q$ increases. In the case of oil production, $Q$ represents the cumulative oil produced (e.g. in barrels), $\dot{Q}$ represents the production rate (e.g. in barrels per day), and $Q_{\text {tot }}$ represents the total recoverable oil that ultimately will be produced from a reservoir or, more broadly, from an oil producing region. $Q=Q_{t o t} /\left(1+\exp \left(r\left(t_{m}-t\right)\right)\right)$ is the solution to logistic differential equation, where $t_{m}$ is the midpoint time (i.e. the time at which $Q$ has grown to $Q_{t o t} / 2$ ). The 
derivative of this function is given by $\dot{Q}=r Q_{t o t} \exp \left(r\left(t_{m}-t\right)\right) /\left(1+\exp \left(r\left(t_{m}-t\right)\right)\right)^{2}$, which is a bell-shaped function that peaks at the midpoint time $t_{m}$.

In 1956, Hubbert fit data for the U.S. oil production rate in the lower-48 states with a curve having the functional form of $\dot{Q}$ and correctly extrapolated the production rate to its peak in 1970 [1]. A key implication of Hubbert's use of logistic growth to empirically model oil production is that the 1970 peak occurred when about half the total recoverable oil in the lower- 48 states had been produced. In other words, according to the logistic growth model, the peak in the oil production rate signifies the midpoint of production. Is there an underlying physical justification for this decline from the midpoint model beyond the empirical observation that production in many oilfields and regions appears to obey logistic growth? In the next section we develop a highly simplified model that offers a physical basis for the observed decline from the midpoint behaviour.

\section{The toy model}

Our toy model is intended to capture essential physical aspects of oil recovery, which is a pressure-driven process ordinarily limited by a drop in pressure and infiltration by impurities such as water or gas. We treat an oil reservoir as a sealed container filled with oil (liquid petroleum), which is assumed to be incompressible, and an ideal gas at pressure $P_{g}(t)$ and volume $V_{g}(t)$. We assume the initial pressure of the gas $P_{g 0}$ is much greater than atmospheric pressure $P_{a t m}$. A pipe, which has a small volume compared to the volume of oil, extends into the container with a cross-sectional area $A(t)$, which represents the total area of all wells and, for simplicity, is assumed to be a continuous function of time. Expanding gas forces liquid oil out of the container through the pipe. We assume the flow of oil obeys Bernoulli's principle, $v^{2}=2\left(P_{g}-\left(P_{a t m}+\rho g h\right)\right) / \rho$, where $v(t)$ is the velocity of exiting liquid oil, $\rho$ is the density of oil, and $h$ is the height of the column of oil in the pipe. We also assume the gas expands slowly and thus isothermally, which implies $P_{g 0} V_{g 0}=P_{g} V_{g}=P_{g}\left(V_{g 0}+V_{e}\right)$, where $V_{e}(t)$ is the volume of oil that has exited the pipe (i.e. the oil produced). Combining these equations and rearranging gives

$$
V_{e}=\frac{P_{g 0} V_{g 0}}{P_{a t m}^{*}+\rho v^{2} / 2}-V_{g 0},
$$

where $P_{a t m}^{*}=P_{a t m}+\rho g h . \quad$ Differentiating eqn. (1) yields the following expression for the volume per unit time of liquid oil exiting the pipe:

$$
\dot{V}_{e}=-\frac{\rho P_{g 0} V_{g 0} v \dot{v}}{\left(P_{a t m}^{*}+\rho v^{2} / 2\right)^{2}},
$$


where a dot indicates differentiation with respect to time. Combining eqn. (2) with conservation of mass, which requires that $\dot{V}_{e}=A v$, leads to a nonlinear ordinary differential equation for the velocity:

$$
\dot{v}=-\frac{A\left(P_{a t m}^{*}+\rho v^{2} / 2\right)^{2}}{\rho P_{g 0} V_{g 0}} .
$$

Equations (1-3) constitute the toy model. In a real oilfield, the total area of wells and the wellhead pressures are the critical determinants of the production rate. Similarly, in the toy model one must specify the initial pressure and volume of gas and the time-varying area $A$ to solve eqn. (3) for the velocity $v$, which then can be substituted into eqn. (1) and eqn. (2) in order to find the volume $V_{e}$ and flow rate $\dot{V}_{e}$ of exiting oil. The pressure that drives the flow of oil at a real oilfield is often due to a variety of fluids (e.g. water and gas). The assumption in the toy model of a single expanding gas driving the flow is a simplification intended to approximate the pressure-driven aspect of primary oil recovery.

For most plausible choices of $A$, the toy model needs to be solved numerically. However, it is possible to solve the model analytically by making the ansatz $v=v_{m} \exp \left(r\left(t_{m}-t\right) / 2\right)$, where $v_{m}$ is the velocity of oil exiting the pipe at the midpoint time. Then eqn. (3) is solved if

$$
A(t)=\frac{P_{g 0} V_{g 0} \rho r v_{m} e^{r\left(t_{m}-t\right) / 2}}{\left(2 P_{a t m}^{*}+\rho v_{m}^{2} e^{r\left(t_{m}-t\right)}\right)^{2}} .
$$

Substituting the ansatz for $v$ into eqn. (2) yields

$$
\dot{V}_{e}(t)=\frac{P_{g 0} V_{g 0} r e^{r\left(t_{m}-t\right)}}{\left(2 P_{a t m}^{*} / \rho v_{m}^{2}+e^{r\left(t_{m}-t\right)}\right)^{2}} .
$$

$\dot{V}_{e}$ as given by eqn. (5) has the same functional form as the derivative of the solution to the logistic differential equation, which is the bell-shaped curve that Hubbert used to predict U.S. peak oil production. Compare $\dot{V}_{e}$ in eqn. (5) to the expression for $\dot{Q}$ in the previous section to see that their functional forms are the same. Self-consistency requires that conservation of mass $\dot{V}_{e}=A v$ is still satisfied and this is easily verified. Alternatively, one can substitute the ansatz into eqn. (1) and differentiate to arrive at eqn. (2).

Figure 1 shows the resulting generic curves for $A$ and $\dot{V}_{e}$ when the same value of parameters in eqn. (4) and eqn. (5) are used. The functions $A$ and $\dot{V}_{e}$ are similar bell-shaped curves with the peak for $A$ occurring after the peak for $\dot{V}_{e}$. In real oilfields, the number of active wells, to which $A$ is roughly proportional, increases as production ramps up and typically reaches a plateau as 


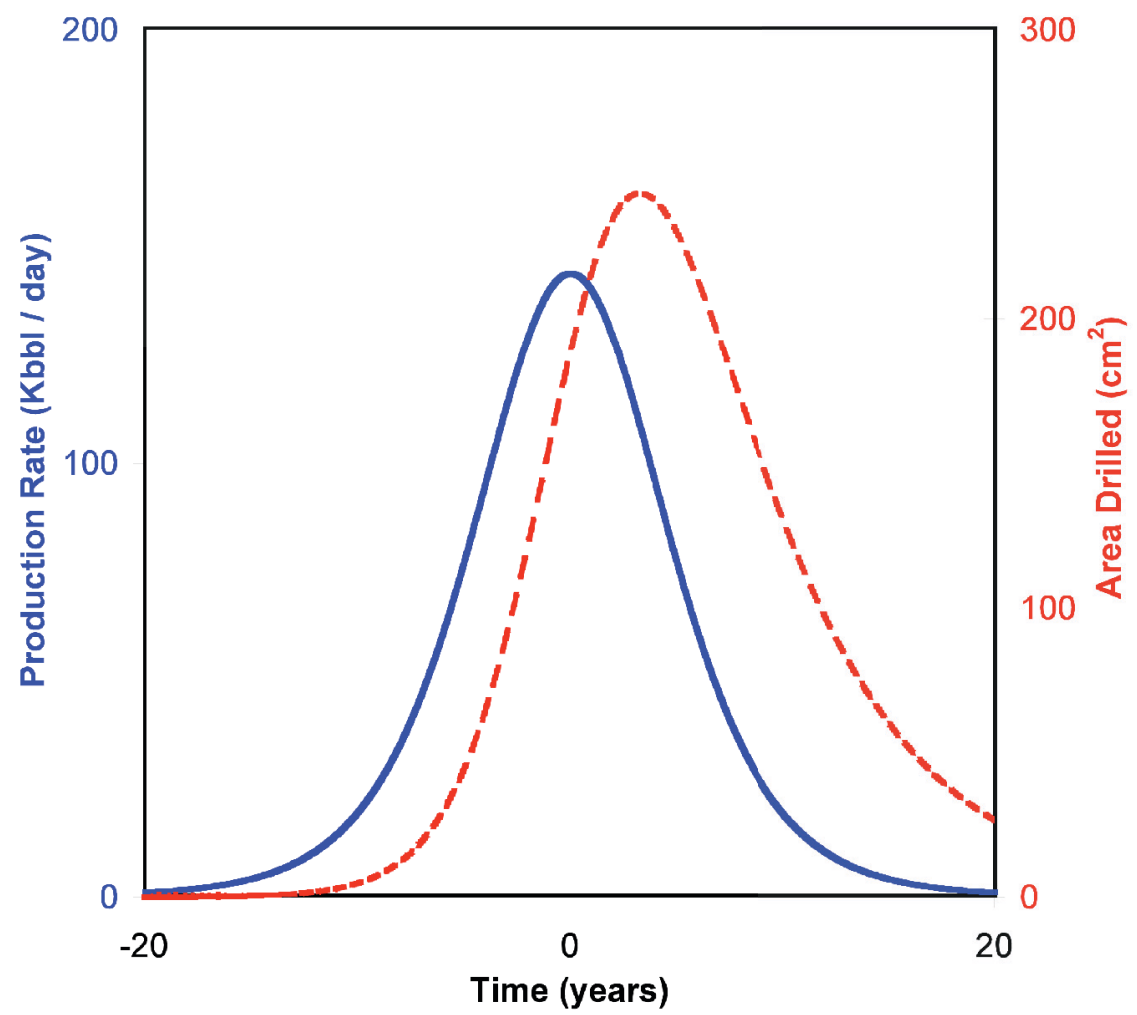

Figure 1: $\quad$ A plot of production rate $\dot{V}_{e}$ (solid curve) and area of active wells $A$ (dashed curve) versus time for typical parameters, showing that the two functions are bell-shaped and $A$ peaks after $\dot{V}_{e}$.

a field matures. However, the economics of diminishing returns dictates that the number of active wells must eventually decrease to zero as production tails off and wells with low production are shut down. Thus, a bell-shaped curve for the function $A$ is plausible. Moreover, a consideration of historical oil production data also makes it plausible that the peak for the number of wells occurs after the peak for the production rate in real oilfields (i.e. $A$ peaks after $\dot{V}_{e}$ ). We have compiled historical oil production data from issues of the Oil \& Gas Journal dating back to 1973 for a large number of oilfields around the world [4]. Such data consistently indicates the number of active wells peaks after the production rate peaks, as can be seen in fig. 2 , which shows the production rate and number of active wells for oilfields on four continents. Indeed, it turns out the toy model predicts this counter-intuitive result for any bell-shaped $A$. This prediction follows from conservation of mass, $\dot{V}_{e}=A v$, and the simple physical assumption in the toy model that the velocity of oil exiting a well is monotonically decreasing over time as oilfield pressure drops, i.e. $\dot{v}<0$. At Hubbert's peak, 
which occurs when $\ddot{V}=A \dot{v}+\dot{A} v=0, \dot{A}=-A \dot{v} / v$ which implies $\dot{A}>0$ (since $A$ and $v$ are intrinsically positive) and therefore $A$ has not yet peaked. This general result, based on simple physical reasoning, explains the data in fig. 2 and other historical data for which the number of active wells is increasing even as the production rate is declining.

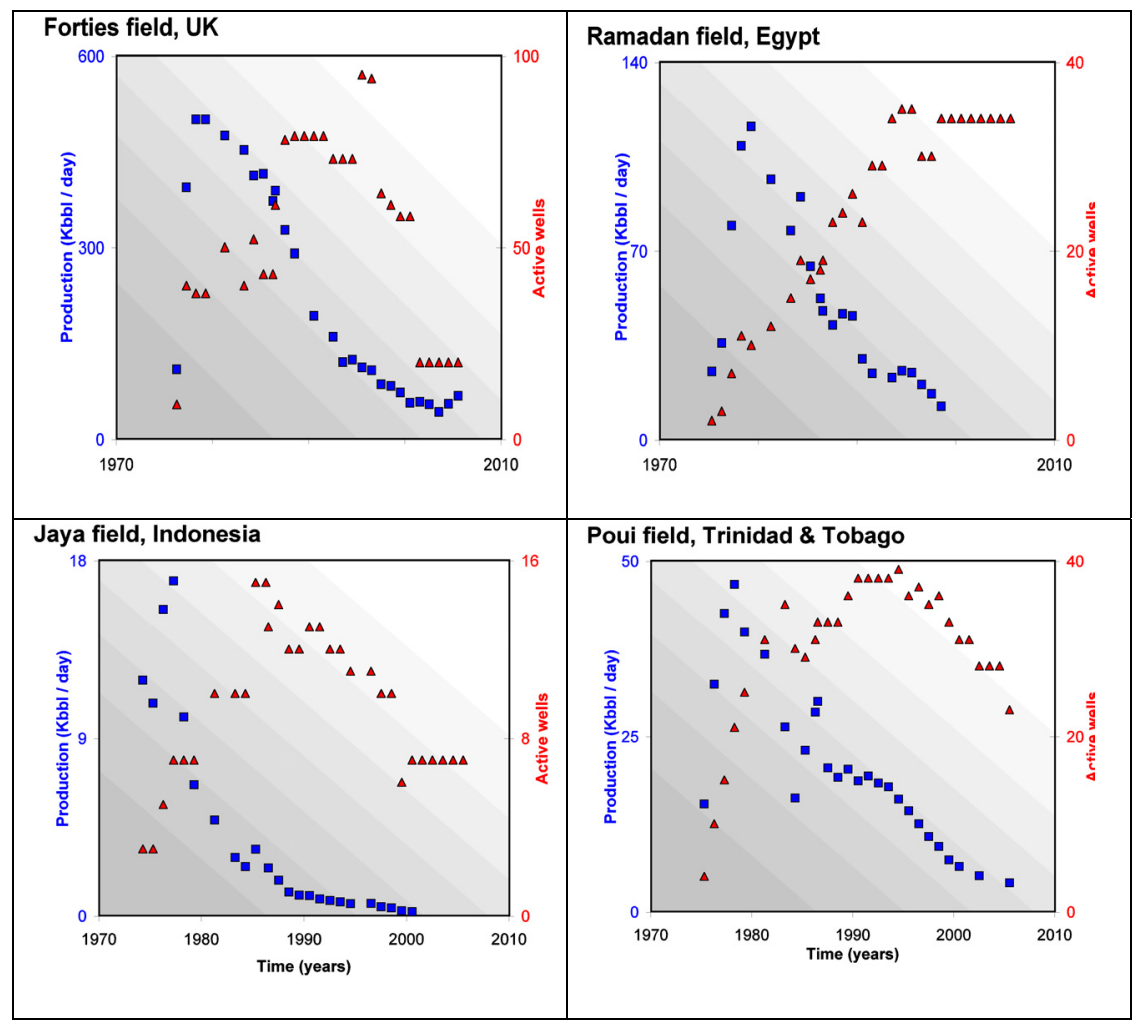

Figure 2: The oil production rate (squares) and the number of active wells (triangles) versus time for oilfields from four continents.

\section{Conclusion}

We have developed a toy model, based on essential physical aspects of oil production, which helps explain Hubbert's peak. We have shown analytically, under plausible assumptions about how the cross-sectional area of active wells at real oilfields typically varies over time, the model is equivalent to the logistic differential equation. We also have shown analytically, contrary to intuition, the number of active wells at an oilfield always peaks after the production rate peaks. We presented historical data from major oilfields on four continents that 
confirm this counter-intuitive consequence of the model. Thus, our highly simplified physical model quite naturally gives rise to Hubbert's peak, i.e. Hubbert's decline from the midpoint empirical model of oil production.

\section{Acknowledgements}

The authors would like to thank D. de Graaf for suggesting this approach to modelling oil production, Steve Strogatz and Michael Boardman for useful consultations, and Roger Blanchard for suggesting a possible source of data.

\section{References}

[1] Hubbert, M.K. Nuclear energy and fossil fuels. Am. Pet. Inst. Div. of Prod. Spring Meeting, Publication No. 95, Shell Development Company: Houston, pp. 1-40, 1956.

[2] Deffeyes K.S., Hubbert's Peak: the Impending World Oil Shortage, Princeton University Press: Princeton, 2001.

[3] Hubbert, M.K., The world's evolving energy system. Am. J. Phys. 49, pp. 1007-1029, 1981.

[4] Abrams, D.M. \& Wiener, R.J., in preparation. 